\title{
Orbit determination of asteroid 2002 KM6
}

\author{
Hyunjin Lee, Catherine Lu, Carter Moyer
}

\begin{abstract}
The premise of this research is to determine the orbit of the asteroid 997952002 KM6 and predict its trajectory far in the future.

Asteroids' orbits model ellipses and can thus be described by six orbital elements: the semimajor axis, eccentricity, inclination, longitude of the ascending node, argument of perihelion, and the mean anomaly. Using Python, our team programmed a series of orbital determination codes, implementing the Method of Gauss to generate the orbital elements with reasonable level of error. This required gathering data over at least three observations. We then compared these elements to those generated by JPL Horizons to ensure they were reasonable.

Our team used the numerical integration program Swift to determine long-term orbital patterns over the next 50 million years. Simulations of 60 different clones of 2002 KM6, randomly sampled from a Gaussian distribution, revealed that the majority of asteroid particles will either get too far or too close to the Sun after 50 million years. A small percentage of them, however, will maintain a stable orbit.
\end{abstract}

\section{Introduction}

The Solar System is one of the millions of planetary systems residing the Milky Way galaxy. It orbits the center of the Milky Way at around $828,000 \mathrm{~km} / \mathrm{hr}$ and is made up of eight planets, many with their own moons, thousands of comets, and millions of asteroids, with the latter being our primary focus [3]. Comets tend to come from the outer reaches of the solar system, covered in ice and orbiting the sun in highly elliptical orbits. They are well known for the tails that appear as they approach the sun and some of the ice along their surface heats up. Finally, we have the asteroids.

Asteroids range in size from a few meters to hundreds of kilometers across, with the largest inner solar system asteroid, Ceres, spanning over 900 kilometers. Most asteroids, however, are substantially smaller. They are located between the orbits of Mars and Jupiter in the Asteroid Belt, and many Trojan asteroids lie in Jupiter's L4 and L5 Lagrange points 60 degrees ahead and behind the planet in its orbit. However, for the purposes of our project, we are looking at 99795 2002 KM6, a Mars-crossing asteroid, which is similar to a near-Earth asteroid. It is particularly important to observe these asteroids as they may pose a threat to Earth. As a matter of fact, 11 percent of near-Earth asteroids are classified as potentially hazardous objects (PHO).

Furthermore, learning more about asteroids can increase our knowledge about the origin of our solar system. Employing the help of astronomers, professionals and amateurs alike, search campaigns for asteroids are launched annually. For example, the All-Sri Lanka Asteroid Search 


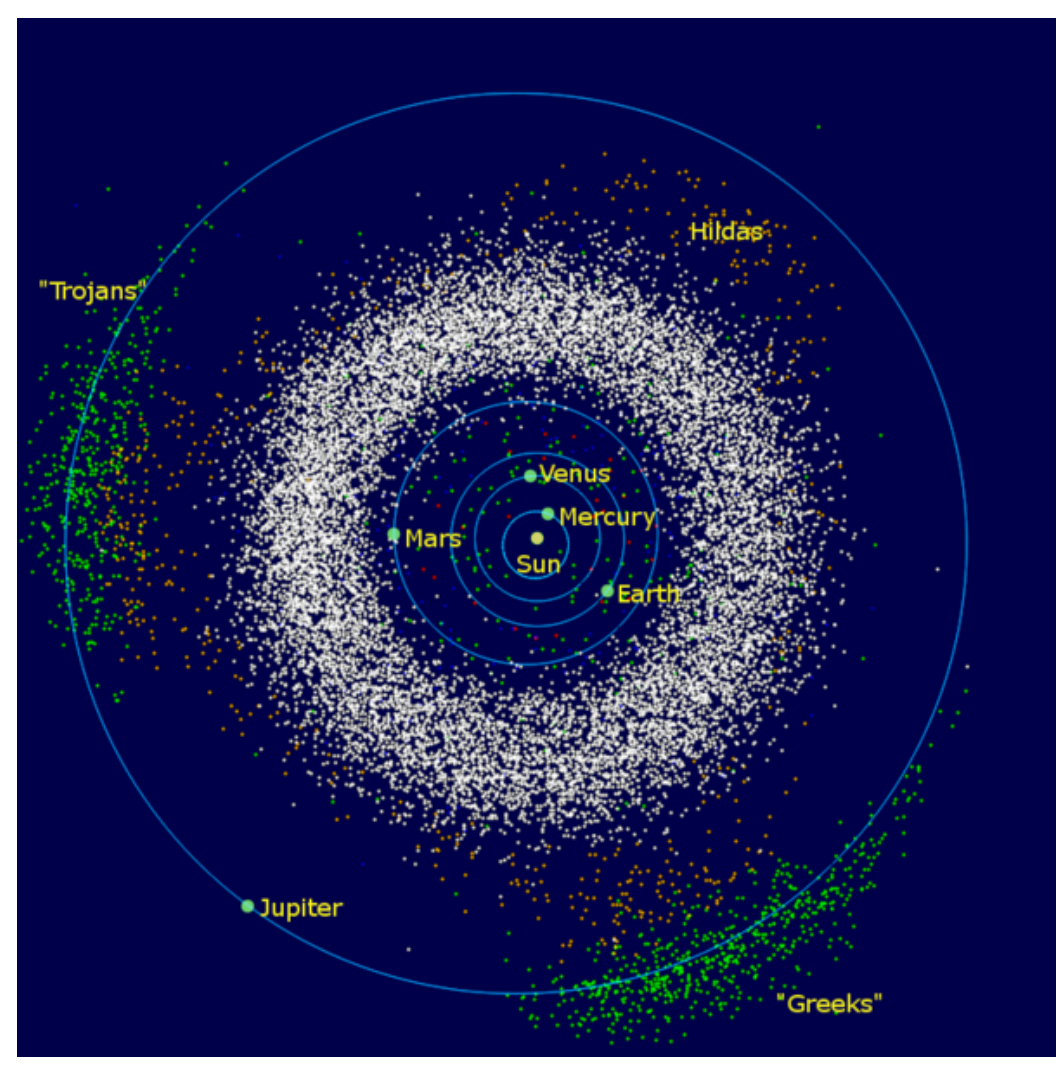

Figure 1: Map of the locations of asteroids

Campaign (ASASC) has discovered more than 228 new main-belt asteroids to date, which may be used as subjects of study in the future.

\subsection{Orbital Elements and Gauss's Method}

The orbits of many asteroids are largely known due in part to these search campaigns, as is the case for asteroid 2002 KM6. The trajectory of the asteroid can be characterized by the position vector, $\overrightarrow{r_{i}}$ and the velocity vector, $\dot{\overrightarrow{r_{i}}}$. The determination of six parameters can specify the unique orbit of an asteroid: the shape, size, orientation, as well as the position of the asteroid's orbit at any specified time [2]. These six parameters, illustrated in Figure 2, are called the orbital elements.

Asteroids follow a Keplerian orbital pattern, and in the case of 2002 KM6, its orbital motion traces out an elliptical pattern. Therefore, two elements are needed in order to describe the size and shape of the elliptical orbit. The first element, Semi-major Axis (a), measures the size of the elliptical orbit and is measured in astronomical units (AU). An ellipse can be represented by the equation below:

$$
\frac{x^{2}}{a^{2}}+\frac{y^{2}}{b^{2}}=1
$$

where $a=$ semi-major axis, or half of the sum of the periapsis and apoapsis distances of the orbit, and $b=$ semi-minor axis. The second orbital element, Eccentricity $(e)$, measures the elongation of 


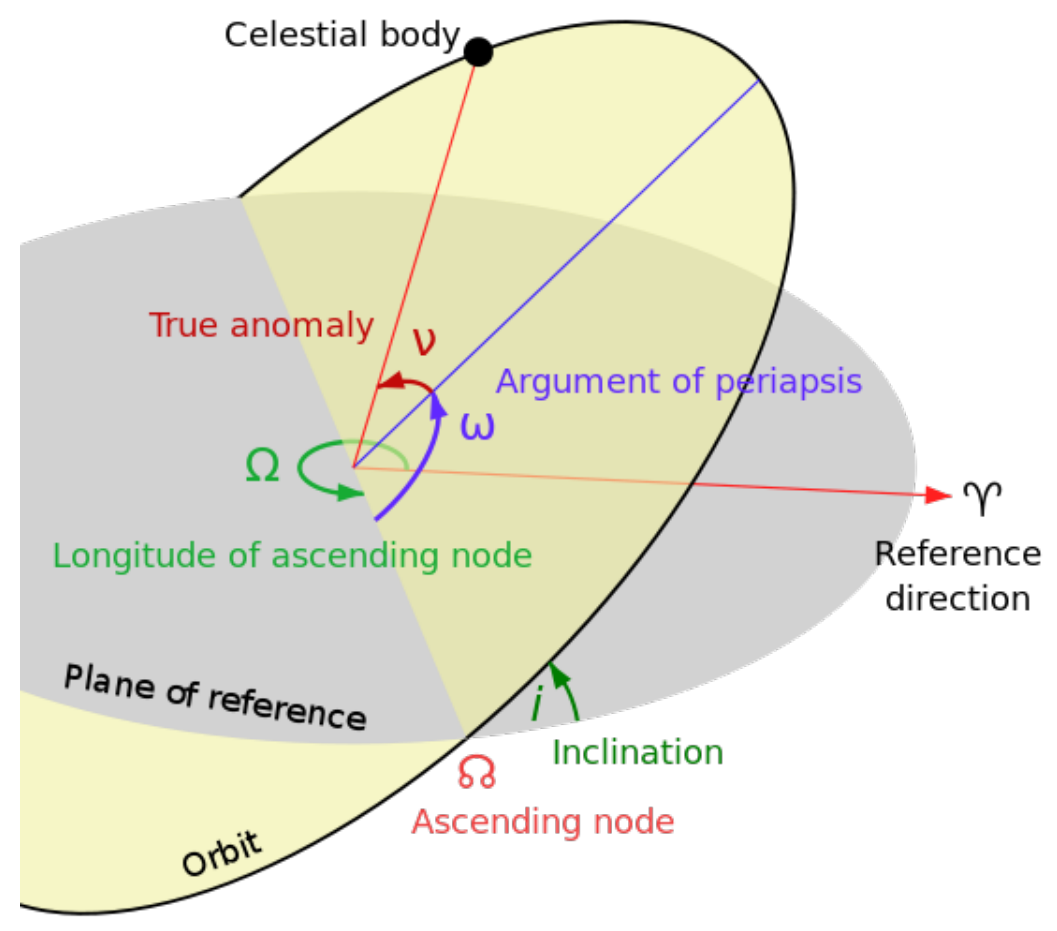

Figure 2: Summary of the six orbital elements

the elliptical path. $e$ can also be expressed as:

$$
e=\sqrt{1-\left(\frac{b}{a}\right)^{2}}
$$

Since an asteroid's orbital plane is not the same as the ecliptic plane, three elements are needed to orient the elliptical path within the orbital plane and express it with respect to the ecliptic. The third orbital element is the Angle of Inclination ( $i$ ), which is the angle between the asteroid's orbital plane and the Earth's ecliptic plane. This value is most commonly reported in degrees. The fourth orbital element is the Longitude of the Ascending Node $(\Omega)$. As stated earlier, the orbital plane and the ecliptic plane are not in the same orientation, and as a result, they intersect at two points called nodes. The ascending node is the point where the asteroid moves north through the ecliptic plane. $\Omega$, reported in degrees, measures the angle between the point of the Vernal Equinox and the ascending node. The fifth orbital element, Argument of Perihelion ( $\omega)$, also reported in degrees, marks the point of the closest approach to the Sun and is the angle between the ascending node and the perihelion, the point of an asteroid's orbit where it is the closest to the Sun. The last orbital element, the Mean Anomaly $(M)$, is a time dependent measure which places the asteroid at a specific location in its orbit. $M$ is also referred to as the Time of Last Perihelion Passage (T). $M$ gives the angular position, in degrees, of the asteroid if the asteroid was in a circular orbit as seen from the center of an ellipse. The value of $M$ can be found by running the Newton's Method of 
Approximation on Kepler's Equation, an equation relating the eccentric anomaly, E and the orbital element $e$.

$$
M=E-e \sin E
$$

In order to determine the orbit of 2002 KM6 we implemented the Method of Gauss for orbit determination. Given the values of $t_{i}$, (times of the observations), $\alpha_{i}$, (RA) and the $\beta_{i}$, (DEC), we can derive the values of $\overrightarrow{r_{i}}$ and $\dot{\overrightarrow{r_{i}}}$ from the Sun to the asteroid. Although the Method of Gauss is a two-body approximation, accounting only for the asteroid and the Sun, it is sufficient for approximating the orbit of our asteroid for a short range of time as the mass of the Sun is more than 1000 times any other body in our solar system. For the approximation to succeed, data from three different observations, roughly evenly spaced, are needed. Because the data is used to approximate deviations from the great circular motion in the night sky, the observations must not be spaced too close together.

\section{Observations and image processing}

\subsection{Data Acquisition}

The observations took place at the University of Colorado Boulder's Sommers-Bausch Observatory, located at $254.7375^{\circ} \mathrm{E}, 40.004^{\circ} \mathrm{N}$, and $1656.6 \mathrm{~m}$ in altitude. The observations of the asteroid took place in the 4:45-6:00 and 6:00-7:15 UTC time range. We used 20-inch PlaneWave telescopes, with CDK20, focal ratio of f/6.8, focal length $3.454 \mathrm{~m}$, SBIG STF-8300M camera with 5.4 pixels, and $52 \mathrm{~mm}$ field of view to take images of our asteroid. The SkyX Professional edition software was used to point the telescope and visualize the images. Table 1 displays the seven observation days as well as the observing time frames and telescope used (East or West).

Table 1: Table displaying asteroid observation days and telescope utilized

\begin{tabular}{lcc}
\hline Observing day (UTC) & Observing time (UTC) & Telescope \\
\hline $2019-06-27$ & $6: 00-7: 15$ & West \\
$2019-06-29$ & $6: 00-7: 15$ & West \\
$2019-07-03$ & $4: 45-6: 00$ & West \\
$2019-07-07$ & $6: 00-7: 15$ & East \\
$2019-07-15$ & $6: 00-7: 15$ & West \\
$2019-07-17$ & $4: 45-6: 00$ & West \\
$2019-07-19$ & $6: 00-7: 15$ & East \\
\hline
\end{tabular}

While we had seven observation dates, not all days provided the ideal sky conditions or other variables to produce clear images of our asteroid. The following table displays the observing dates and details $(\mathrm{RA}=$ right ascension, $\mathrm{DEC}=$ declination $)$ : 


\begin{tabular}{|c|c|c|}
\hline Date \& Time (UTC) & Images taken & Observation details \\
\hline $\begin{array}{l}2019-06-27 \\
6: 00-7: 15\end{array}$ & 10 lights, 9 darks & $\begin{array}{l}\text { Exposure time: } 65 \text { seconds. } \\
\text { Sky was very clear. } \\
\text { We did not record our focus star or its location. }\end{array}$ \\
\hline $\begin{array}{l}2019-06-29 \\
6: 00-7: 15\end{array}$ & 10 lights, 9 darks & $\begin{array}{l}\text { Exposure time: } 65 \text { seconds. } \\
\text { Focus star: Izar } \\
(\mathrm{RA}=14: 45: 50.283, \mathrm{DEC}=26: 59: 49.533, \mathrm{APmag}=2.35) \\
\text { Sky was initially very cloudy as the } \\
\text { observation was after a storm. } \\
\text { Processing the images from this observation } \\
\text { failed to reveal the asteroid. }\end{array}$ \\
\hline $\begin{array}{l}2019-07-03 \\
4: 45-6: 00\end{array}$ & 10 lights, 9 darks & $\begin{array}{l}\text { Exposure time: } 65 \text { seconds. } \\
\text { Focus star: Unukalkai } \\
(15: 45: 13.6317,0.6: 22: 04.493, \text { APmag }=2.63) \\
\text { Sky was very clear but storm was coming in } \\
\text { toward the end of the observation. }\end{array}$ \\
\hline $\begin{array}{l}2019-07-07 \\
6: 00-7: 15\end{array}$ & 10 lights, 9 darks & $\begin{array}{l}\text { Exposure time: } 73 \text { seconds. } \\
\text { Focus star: HIP } 86263 \\
(17: 38: 42.59,-15: 27: 28, \text { APmag }=3.519) \\
\text { Sky was slightly cloudy but still clear } \\
\text { enough for stars to be visible. }\end{array}$ \\
\hline $\begin{array}{l}2019-07-15 \\
6: 00-7: 15\end{array}$ & 10 lights, 5 darks & $\begin{array}{l}\text { Exposure time: } 70 \text { seconds. } \\
\text { Focus star: HIP } 94141 \\
(19: 10: 55.7415,-20: 59: 23.148, \text { APmag }=2.88) \\
\text { Sky was clear. } \\
\text { The West telescope had a slight pointing error. } \\
\text { To account for this, } \\
13 \text { arc seconds was subtracted from the RA } \\
\text { and } 1 \text { arcminute was added to the DEC. } \\
\text { The images from this observation were slightly blurry, } \\
\text { but the asteroid was found after processing. }\end{array}$ \\
\hline $\begin{array}{l}2019-07-17 \\
4: 45-6: 00\end{array}$ & 10 lights, 5 darks & $\begin{array}{l}\text { Exposure time: } 70 \text { seconds. } \\
\text { Focus star: Sabik } \\
(17: 11: 30.0394,-15: 44: 48.439, \text { APmag }=2.43) \\
\text { The moon was very bright so the light reflecting } \\
\text { from the moon on the clouds may have affected } \\
\text { the quality of the images. } \\
\text { Processing the images from this night } \\
\text { failed to reveal the asteroid. }\end{array}$ \\
\hline $\begin{array}{l}2019-07-19 \\
6: 00-7: 15\end{array}$ & 10 lights, 5 darks & $\begin{array}{l}\text { Exposure time: } 70 \text { seconds. } \\
\text { Focus star: Kaus Australis } \\
(18: 25: 28.468,-34: 22: 20.586, \text { APmag }=1.79) \\
\text { The sky was fairly clear. }\end{array}$ \\
\hline
\end{tabular}


On the day of every observation, an ephemeris for the asteroid during the observing time window was generated using JPL Horizons. Before taking our series of asteroid images, flat frames (normally exposed images with the entire field of view) were taken. These were necessary in order to remove dust donuts and eliminate the effects of vignetting during processing. For every observation, five flat frames were taken. After flat frames, we focused the telescope cameras using focus stars near the meridian and with magnitudes APmag $=2$ to 4. A Bahtinov Mask was placed on the front of the telescope and series of photos of the focus star were taken until the diffraction pattern of the star images had a central spike bisecting an "X" pattern, indicating proper focus. The telescope was then slewed to the asteroid coordinates predicted by the JPL Horizons ephemeris. Test images were taken of the asteroid to make sure the star field in the image matched the one printed from the DS9 database. When the star fields were matched, we began series of images of the asteroid (alternating from light exposures and dark exposures). Because asteroid 2002 KM6 has an apparent magnitude around 15, an exposure time of 65 seconds was appropriate. We made sure to keep the camera temperatures stable in order to reduce the effects of thermal noise during the exposure. After images were taken, the raw light-exposed images were processed with the dark exposures and flat frames in AstroImageJ (AIJ). The dark frames and flat frames were subtracted from the light frames in order to isolate the signal frame, correct for other grain variations, and calibrate science images, resulting in a clean plate for further analysis.

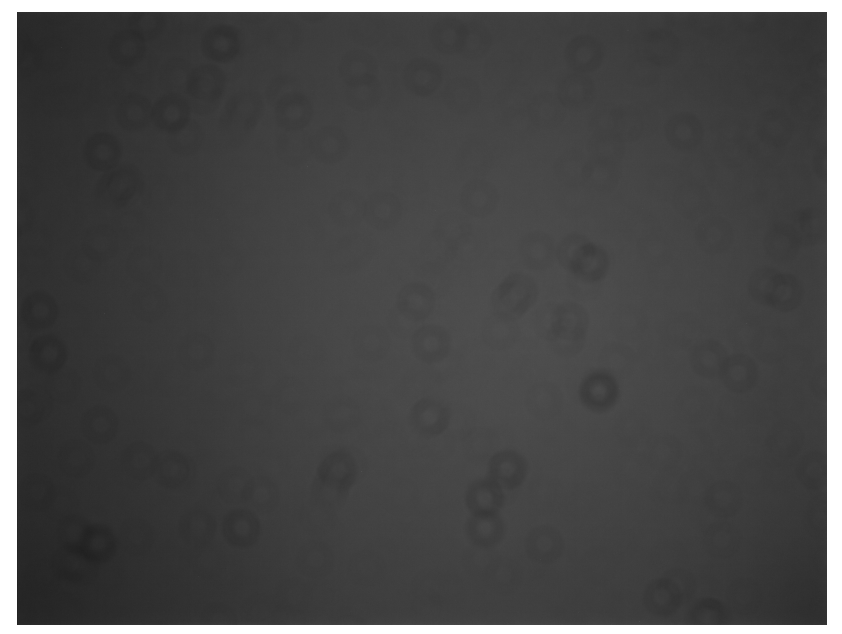

Figure 3: Flat Frame from the July 3rd observation, displayed on AstroImageJ

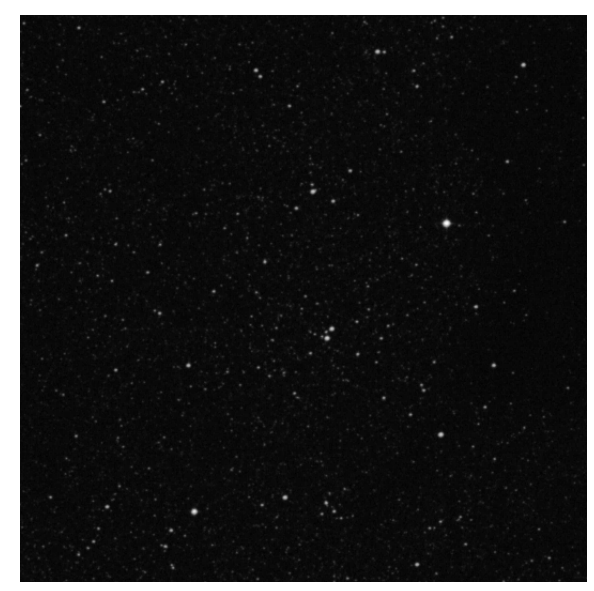

Figure 4: Star field generated with the DS9 software on the July 15th observation date

\subsection{Image Reduction}

The process of image reduction includes astrometry, which we performed on our calibrated science images using the AstroImageJ and SAO DS9 softwares.

Astrometry allows for the precise measurements of the positions and movements of celestial objects. For our purposes, we used astrometric techniques to calculate the RA and DEC of the asteroid as well as those of our reference stars. Our chosen reference stars are known, cataloged stars that are not saturated in the asteroid images (photon counts; 45,000). The magnitude of the 
reference stars should also have the same visual magnitudes (Vmag) as those listed in the DS9 database.

To select our reference stars, we opened the SAO DS9 software and using the ESO-DSS image server, the RA and DEC values obtained from the observation or JPL Horizons, and using a $15 \mathrm{x}$ 15 arc minutes field of view, we generated star charts for the observation. After that, the USNO UCAC4 optical catalog was chosen and brightness thresholds were set in order to identify easily identifiable stars in the star charts. Once we generated DS9 star charts, we subsequently opened processed light images (in the FITS file format) from an observation date with AIJ.

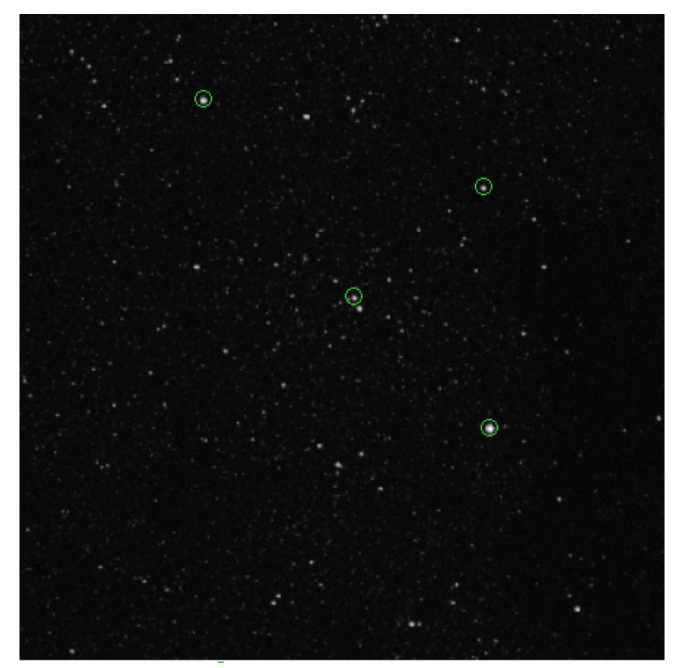

Figure 5: Identified stars generated from the USNO UCAC4 optical catalog

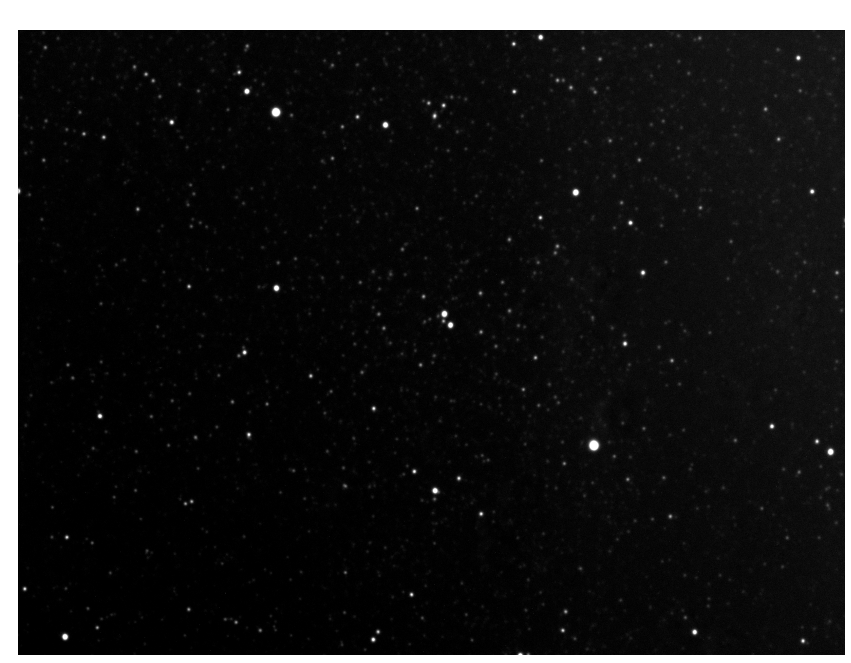

Figure 6: Reduced image viewed on AstroImageJ from the July 17 th observation

In order to make sure the images from the chosen observation are able to be used for astrometry, we had to locate the asteroid in the images. We blinked through the sequences of images to identify a moving object, then performed astrometric calibration with AIJ's plate solve function. If we could not find a moving object, the observation plates were discarded, as well as if the object was too obscured by surrounding stars. Lastly, we determined the centroid of the reference stars and asteroid. We set the inner radius of the mouse aperture to capture all the photons from the bright pixels at its center and the annulus formed by the two radii to capture the background (sky) photons. Once set, AIJ determined the image coordinates and the RA and DEC of the centroid of the selected object. These data were exported in order to run error analysis, as will be detailed in a later section.

\subsection{Determination of Errors in Astrometry}

The plate solve function in AIJ, which identifies stars and returns the RA and DEC (position) of target bodies and the star field, does not provide any information about the uncertainty of its results. Since the plate solve is an integral part of our image reduction process, our team completed checks in order to ensure the reliability of the AIJ plate solve results. We performed two independent checks: one that compared the RA and DEC values of the asteroid determined by AIJ with those obtained from a handwritten least squares plate reduction (LSPR) code, and one that compared the 
known RA and DEC of several reference stars determined in DS9 with the corresponding positions given by the AIJ plate solve.

\subsubsection{Manual plate solve using LSPR}

We coded a Python LSPR program that, given the RA and DEC for an arbitrary number of reference stars as well as the $\mathrm{x}$ and $\mathrm{y}$ coordinates of both the reference stars and the target asteroid, (determined from CCD), can determine the RA and DEC of the asteroid. The program utilizes linear transformations to relate the plate coordinates $(\mathrm{x}, \mathrm{y})$ and the celestial coordinates $(\alpha, \beta)$ to the RA and DEC. We used the following transformations, (where the a's and b's are plate coefficients), to solve for the RA and DEC.

$$
\begin{gathered}
\alpha=b_{1}+a_{11} x+a_{12} y \\
\beta=b_{2}+a_{21} x+a_{2} y
\end{gathered}
$$

However, because the solutions for RA and DEC are prone to measurement errors, we implemented a least-squares procedure to find the best-fit linear model for the data. This procedure allows for minimization of the sum of the squares of the distances between the estimates and the true values.

For our test, we chose a reduced image plate from the July 7 th observation with a number clean and unsaturated stars. After making sure the star field matched with the image generated from the DS9 server, we chose 6 reference stars surrounding the asteroid 2002 KM6. The right ascension and the declination of the reference stars were determined and using AIJ, the X, Y coordinates of the asteroid and reference stars were also determined. Afterwards, using the RA, DEC, and the $\mathrm{X}$ and Y coordinates found, our team used the LSPR code to determine the RA and DEC of 2002 KM6 at the time of the July 7th observation. The positions and the RA and DEC of the six reference stars and the asteroid are displayed in the table below.

Table 2: A table displaying the data for the asteroid and the six reference stars

\begin{tabular}{lcccc}
\hline & $\mathrm{X}$ & $\mathrm{Y}$ & $\mathrm{RA}\left({ }^{\circ}\right)$ & $\mathrm{DEC}\left({ }^{\circ}\right)$ \\
\hline Star 1 & 702.28 & 488.0031 & 264.611303 & -25.0046098 \\
Star 2 & 751.9015 & 852.2215 & 264.409387 & -24.9691956 \\
Star 3 & 2052.0308 & 895.0708 & 264.478409 & -24.9658256 \\
Star 4 & 1759.227 & 1095.8338 & 264.507497 & -24.9494675 \\
Star 5 & 837.9692 & 1452.1108 & 264.599696 & -24.918367 \\
Star 6 & 380.9108 & 1716.3477 & 264.645243 & -24.8950537 \\
Asteroid & 1879.6757 & 1526.6528 & - & - \\
\hline
\end{tabular}

\subsubsection{Manual plate solve results}

The result of the LSPR code are displayed in table 3 below. While the coordinates determined through the LSPR code and AIJ differ, the differences do not exceed roughly two uncertainties, which is reasonable. Thus, we conclude that AIJ's plate solve is reliable. These results would likely improve had we used a greater number of reference stars, as our data would then be more robust. 
Table 3: Table displaying the final RA and DEC values of asteroid $2002 \mathrm{KM} 6$ as well as the uncertainties.

\begin{tabular}{lcc}
\hline & RA(hours) & DEC $\left(^{\circ}\right)$ \\
\hline 2002 KM6 & 17.633110 & -24.910161 \\
Uncertainties & 0.000231 & 0.0005 \\
AIJ & 17.633055 & -24.908944 \\
\hline
\end{tabular}

The following formulae were used to find the uncertainties of the RA and DEC values:

$$
\begin{gathered}
\sigma_{\alpha}=\sqrt{\sum_{i=1}^{N} \frac{\left(\alpha_{i, P S}-\alpha_{i}\right)^{2}}{N-3}} \\
\sigma_{\beta}=\sqrt{\sum_{i=1}^{N} \frac{\left(\beta_{i, P S}-\beta i\right)^{2}}{N-3}}
\end{gathered}
$$

\subsubsection{Plate Solve Residuals}

For the second check on AIJ plate solve's reliability, we compared the known RA and DEC (obtained from DS9) of the chosen reference stars with the calibrated values from the AIJ plate solve.

We began by choosing a reduced image from the June 26th observation. After plate solving the reduced image to read the RA and DEC of the star field on AIJ, we compared the image with the corresponding DS9 star field. Since the DS9 image initially had over 200 stars, we applied a series of filters to narrow it down. First, we filtered out stars that did not have a Vmag of 0 or a Vmag less than 13.7 to ensure that they were visible. Additionally, to ensure that we only received stars within our field of view, we restricted the possible RA values to be between 264.17 degrees and 265.95 degrees and the possible DEC values to be between -27.63 degrees and -27.94 degrees. We then obtained the RA and DEC of the 20 remaining stars from DS9's USNO UCAC4 optical catalog. Afterwards, these stars were matched to stars on the processed image on AIJ. From DS9's two lists of RA and DEC and the predicted ones from the AIJ plate solve, we calculated the residuals and other measures of uncertainties.

\subsubsection{Plate Solve Residuals Results}

The residuals of the RA (predicted from AIJ - known from DS9) had a mean of 0.2675 arcseconds and a standard deviation of 0.06853 arcseconds. The residuals of the DEC had a mean of 0.2960 arcseconds and a standard deviation of 0.1466 arcseconds. The results reveal that the mean is zero within a standard deviation of the mean for the DEC residuals. However, for the RA residuals, the mean is four standard deviations away from zero, implying a systematic error in the computation of the RA residuals of about 0.2 arc-seconds (the mean of the residuals). Therefore, while the calculations are reliable for finding the DEC, that is not the case for determining the RA on AIJ. 


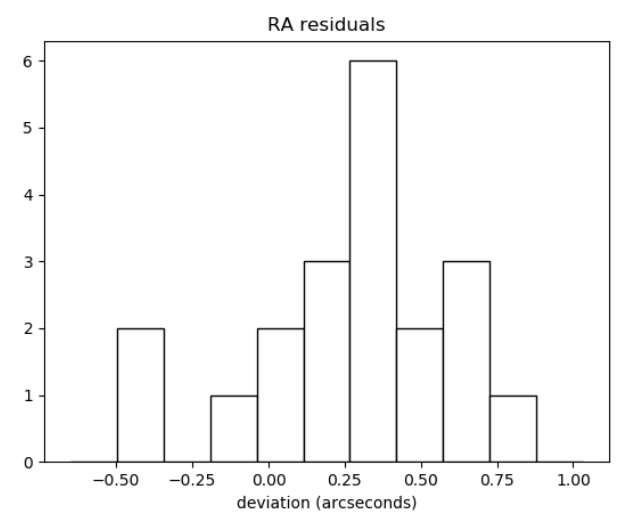

Figure 7: The residuals of the RA of the reference stars between DS9 and AstroImageJ.

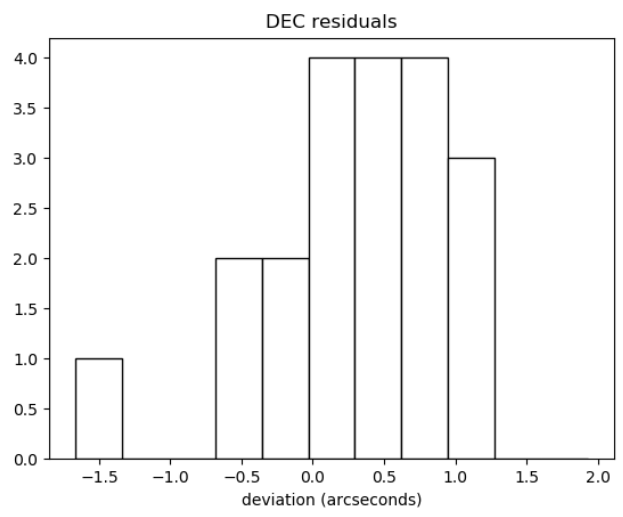

Figure 8: The residuals of the Dec of the reference stars between DS9 and AstroImageJ.

Table 4: The RA (hours) and DEC $\left(^{\circ}\right)$ of the reference stars from DS9 database and AIJ with residuals in arcseconds

\begin{tabular}{lcccccc}
\hline & RA (DS9) & DEC (DS9) & RA (AIJ) & DEC (AIJ) & RA residual & DEC residual \\
\hline Star 1 & 17.66471 & -27.83286 & 17.66472 & -27.83269 & 0.0432 & 0.612 \\
Star 2 & 17.66473 & -27.84415 & 17.66474 & -27.84391 & 0.0295 & 0.886 \\
Star 3 & 17.66518 & -27.87508 & 17.66518 & -27.87487 & -0.00306 & 0.763 \\
Star 4 & 17.66633 & -27.80791 & 17.66634 & -27.80763 & 0.0136 & 1.02 \\
Star 5 & 17.66957 & -27.92528 & 17.66957 & -27.92514 & -0.0250 & 0.536 \\
Star 6 & 17.66990 & -27.86876 & 17.66990 & -27.86854 & -0.000468 & 0.792 \\
Star 7 & 17.67146 & -27.90632 & 17.67145 & -27.90624 & -0.0242 & 0.266 \\
Star 8 & 17.67267 & -27.80570 & 17.67267 & -27.80570 & 0.0162 & 0.0119 \\
Star 9 & 17.67395 & -27.80712 & 17.67396 & -27.80726 & 0.0200 & -0.497 \\
Star 10 & 17.67592 & -27.86369 & 17.67592 & -27.86380 & 0.00346 & -0.407 \\
Star 11 & 17.67681 & -27.83938 & 17.67681 & -27.83981 & 0.0113 & -1.55 \\
Star 12 & 17.66377 & -27.78069 & 17.66378 & -27.78039 & 0.0248 & 1.11 \\
Star 13 & 17.66501 & -27.68119 & 17.66502 & -27.68120 & 0.0187 & -0.0241 \\
Star 14 & 17.66525 & -27.77161 & 17.66526 & -27.77133 & 0.0223 & 1.02 \\
Star 15 & 17.66579 & -27.78733 & 17.66579 & -27.78715 & 0.0209 & 0.630 \\
Star 16 & 17.66616 & -27.67346 & 17.66618 & -27.67347 & 0.0428 & -0.00972 \\
Star 17 & 17.66880 & -27.68036 & 17.66881 & -27.68043 & 0.0515 & -0.250 \\
Star 18 & 17.66908 & -27.79713 & 17.66908 & -27.79697 & 0.0197 & 0.594 \\
Star 19 & 17.66965 & -27.74365 & 17.66966 & -27.74352 & 0.0323 & 0.486 \\
Star 20 & 17.67244 & -27.75644 & 17.67245 & -27.75646 & 0.0396 & -0.0673 \\
\hline
\end{tabular}


Table 5: The residuals of the RA and DEC of the reference stars from DS9 database and AIJ

\begin{tabular}{lcc}
\hline & Mean Residuals [arcseconds] & Standard Deviation [arcseconds] \\
\hline RA & 0.2675 & 0.3065 \\
DEC & 0.2960 & 0.6556 \\
\hline
\end{tabular}

\subsubsection{Error Analysis discussion}

The two studies checking the reliability of the AIJ plate solve involved comparing AIJ's output to that produced by a manually written LSPR program and the USNO UCAC4 database. In the first check, the errors were within two uncertainties, deeming the AIJ plate solve function reliable. The second study checked the residuals of the RA and DEC of reference stars between the AIJ and the DS9 database. Unlike the first residual study, the second revealed that the AIJ plate solve is reliable for the DEC, but not so for the RA calculations. Because the apparent systematic error implied a discrepancy of around 0.2 arc-seconds, caution may be needed regarding the values generated by AIJ for the RA. However, this error could also be attributed to a pointing error on the telescope utilized during this observation.

\subsection{Results}

\begin{tabular}{lcc}
\hline Julian date & RA $\left(^{\circ}\right)$ & DEC $\left(^{\circ}\right)$ \\
\hline 2458661.768 & 265.015 & -27.708969 \\
2458661.785 & 265.014 & -27.70395 \\
2458671.762 & 264.497 & -24.910412 \\
2458671.778 & 264.496 & -24.906182 \\
2458679.761 & 264.639 & -22.782507 \\
2458679.772 & 264.639 & -22.779707 \\
2458683.762 & 264.925 & -21.792642 \\
2458683.774 & 264.926 & -21.790782 \\
\hline
\end{tabular}

Table 6: Table listing the Julian date, RA, and DEC of individual observations

\section{Orbit determination}

\subsection{Methods}

To determine the orbital elements, we need the asteroid's ecliptic position and velocity at a certain time. To accomplish this, we wrote a Python program utilizing the Method of Gauss. Given the RA and DEC of the asteroid on three, roughly evenly separated observation dates and the equatorial Earth-Sun vectors on these dates, the Method of Gauss calculates the asteroid's $\overrightarrow{r_{i}}$ (representing the vector from the Sun to the asteroid) at the time of the middle observation. The algorithm uses a fourth order Taylor series expansion of the $\overrightarrow{r_{i}}$ to generate terms in the $f$ and $g$ 
series, which are then used to approximate the values of $\overrightarrow{r_{i}}$ and $\dot{\vec{r}}$. Our program runs for multiple iterations until the position and velocity vectors converge to common values within $1 \mathrm{E}-10 \mathrm{AU}$, which are then passed to other functions to calculate the orbital elements.

We ran our observation data from July 7 th, July 15th, and July 19th $(2458671.778,2458679.772$, and 2458683.774 JD, respectively) through the program to calculate the orbital elements of our asteroid at the time of our July 15 th observation.

To obtain greater accuracy in our vector output, we also accounted for light-travel times in our Method of Gauss program. Since the distance from the Sommers-Bausch Observatory to the asteroid is non-negligible, the time it takes for light to arrive to SBO is non-negligible as the asteroid has moved in its orbit during that time. In order to solve for the times when the light has left the asteroid, we used the following equation,

$$
t_{i}=t_{o, i}-\frac{\rho_{i}}{c}
$$

where $t_{o, i}$ are the recorded times of the observations, $\rho$ is the Earth-Asteroid vector calculated in iterations of the Method of Gauss algorithm, and $c$ is the speed of light in the unit AU/day.

Additionally, we calculated the uncertainties of each element by performing a Monte Carlo simulation. In this simulation, we randomly sampled RA and DEC values from a Normal distribution with the observed RA and DEC of the asteroid as the mean and the uncertainty of the AIJ plate solve (calculated from our second reliability check) as the standard deviation. We calculated the orbital elements from each sample, then constructed histograms representing the spread of each orbital element. The means of these distributions are our orbital element estimates, and the standard deviations are their uncertainties. Using these orbital element values, we created a visual of the asteroid's orbit around the Sun.

We also checked for consistency in our orbital determination results. To do so, we wrote an ephemeris generation program in Python, which outputs the expected RA and DEC of an object at a certain time given its orbital elements and position in the ecliptic plane at a different time. As inputs, we used our calculated orbital elements at the time of our July 15th observation and computed the expected RA and DEC at the time of our July 19th observation. This output was compared with the actual data recorded from our July 19th observation.

As a two-body approximation, the Method of Gauss fails to account for the gravitational effects from other bodies in the solar system, which is problematic due to 2002 KM6's close proximity to Mars. Thus, we chose to use the numerical integration program Swift to determine the ultimate fate of the asteroid, as it is capable of integrating sets of multiple gravitationally interacting bodies with numerous test particles. In our case, we simulated the orbits of three sets of 20 randomly sampled clones of 2002 KM6 from Gaussian distributions, focusing on the asteroid's interactions with the Sun, Venus, Earth, Mars, Jupiter, and Saturn.

\subsection{Results}


Table 7: Table listing the calculated orbital elements and associated uncertainty

\begin{tabular}{lc}
\hline Orbital element & Value \\
\hline Semi-major axis (AU) & $2.645 \pm 0.004$ \\
Eccentricity & $0.4067 \pm 0.0007$ \\
Inclination (deg) & $9.537 \pm 0.010$ \\
Longitude of the ascending node (deg) & $280.977 \pm 0.006$ \\
Argument of perihelion (deg) & $356.768 \pm 0.093$ \\
Mean anomaly at 2458679.772 JD (deg) & $1.749 \pm 0.037$ \\
\hline
\end{tabular}

Table 8: Table listing the results of the ephemeris self-consistency test

\begin{tabular}{lccc}
\hline & Observed & Generated & Percent Difference \\
\hline RA (deg) & 264.926 & 264.932 & $0.002 \%$ \\
DEC (deg) & -21.784 & -21.791 & $0.032 \%$ \\
\hline
\end{tabular}


Figure 9: Distributions of orbital element values from Monte Carlo simulations
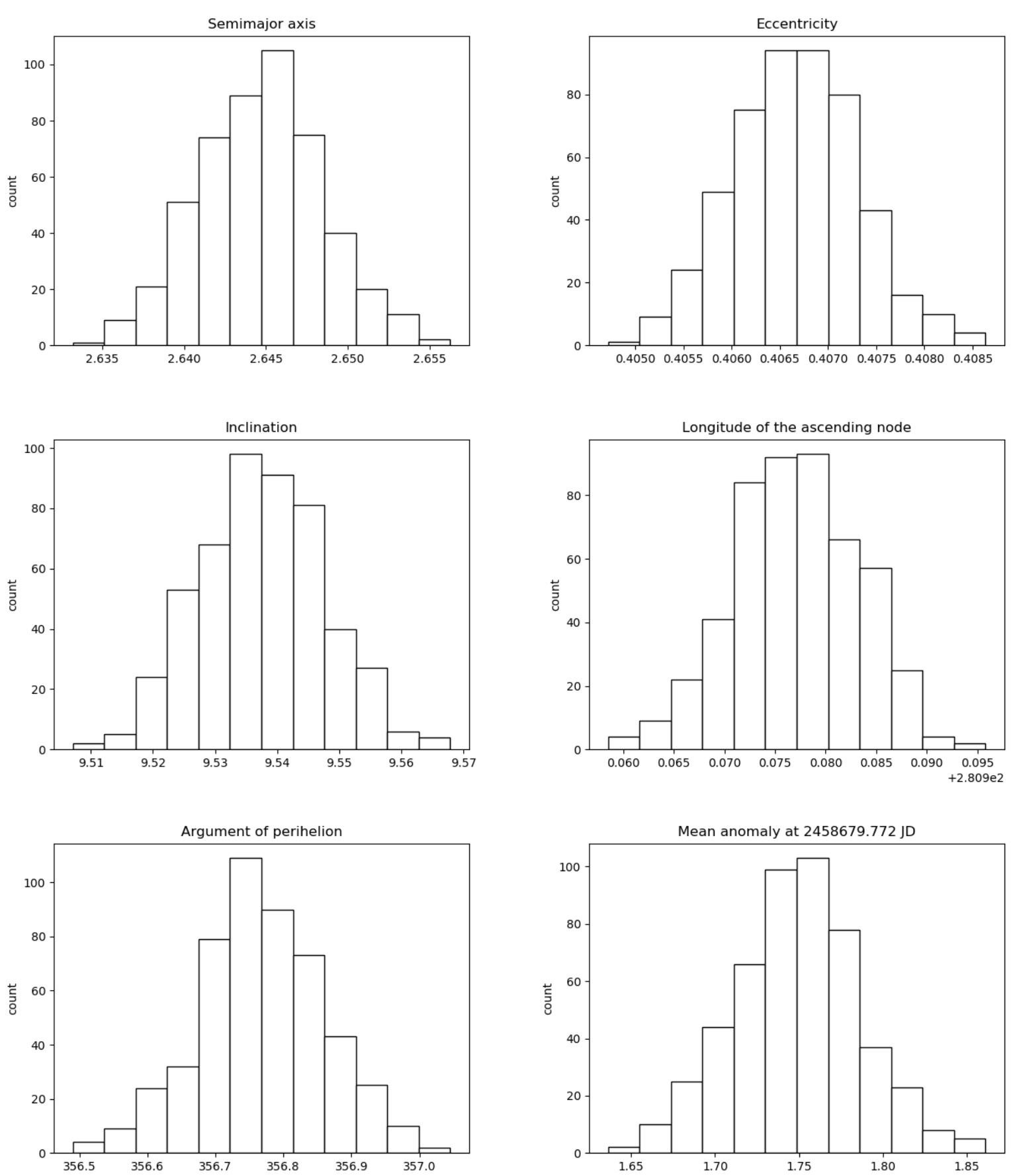
With the results of the Swift numerical Integration, we generated graphs of the aphelion distances (top), semi-major axis (middle), and the perihelion distances (bottom) versus time. Figure 10 displays the graphs of two representative test particles, both showing particles that ejected out of the solar system as their semi-major axis goes on to the upper bounds at the end of the simulation:

Figure 10: Long-term dynamical behavior of 2002 KM6 generated with numerical integration Swift software
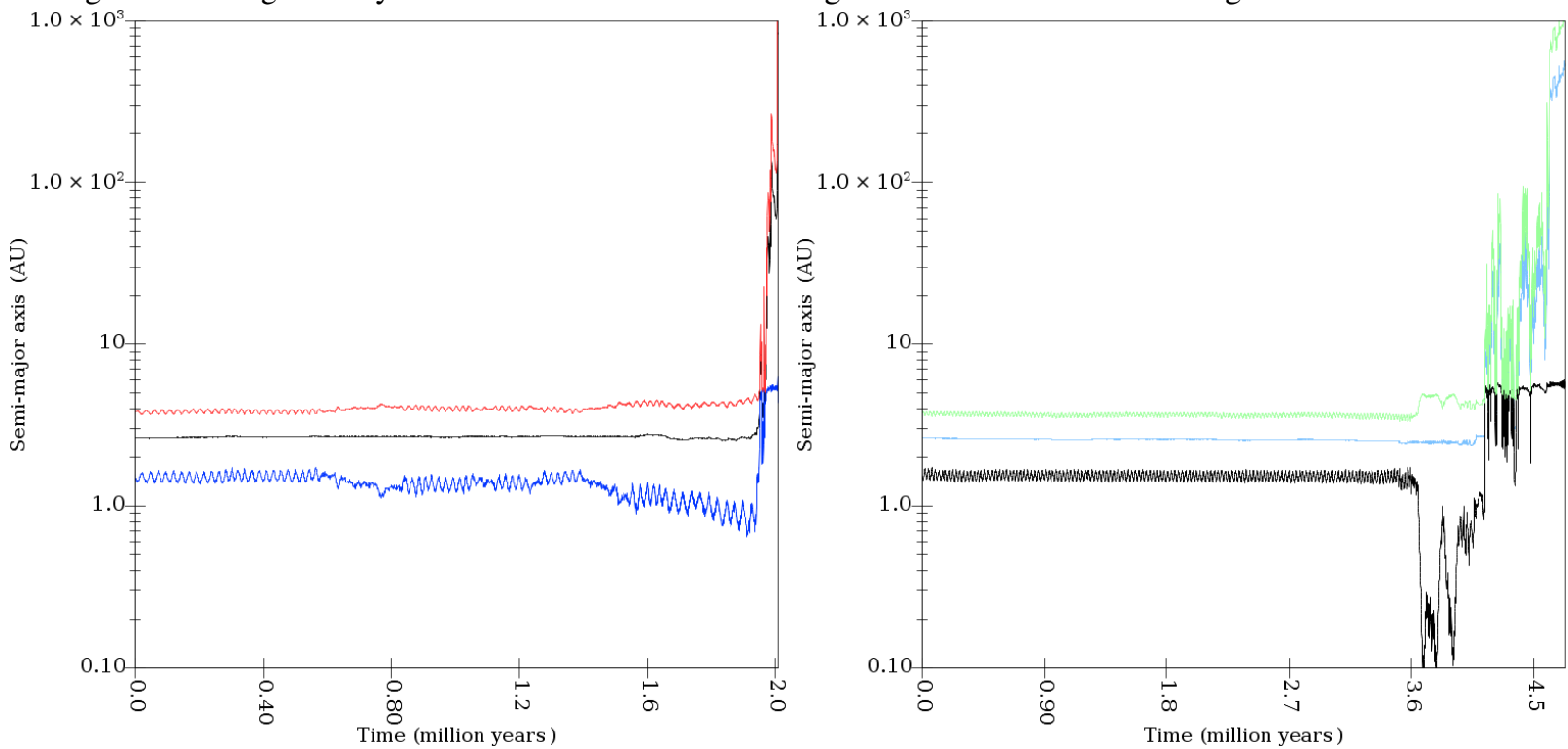

\section{Discussion}

Table 9: Table listing the discrepancies between the generated orbital elements and those of JPL Horizons

\begin{tabular}{lccc}
\hline & Mean of Element & JPL Values & Percent Difference (\%) \\
\hline Semi-major Axis (au) & $2.6447 \pm 0.0039$ & 2.6402 & 0.0017 \\
Eccentricity & $0.4067 \pm 0.0007$ & 0.4059 & 0.0019 \\
Inclination (deg) & $9.5375 \pm 0.0101$ & 9.5271 & 0.0011 \\
Longitude of Ascending Node (deg) & $280.9772 \pm 0.0062$ & 280.9686 & 0.00003 \\
Argument of Periapsis (deg) & $356.7677 \pm 0.0925$ & 356.7195 & 0.0001 \\
Mean Anomaly* (deg) & $1.7487 \pm 0.0375$ & 1.7779 & 0.0166 \\
\hline
\end{tabular}

$*$ at $2458679.772 \mathrm{JD}$ 


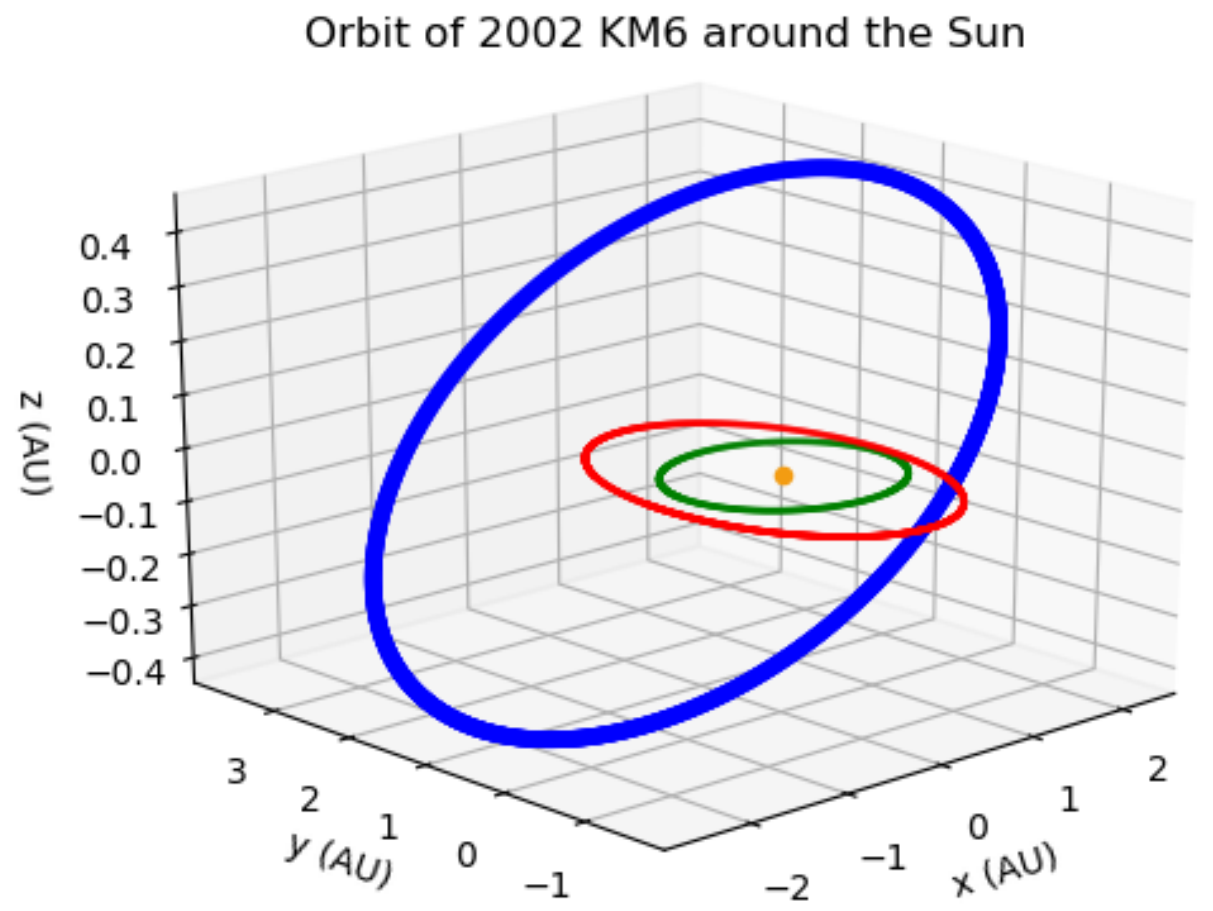

Initially, the three observation times chosen for analysis were July 2nd, July 7 th and July 15 th. However, for this data set, the Method of Gauss failed to return any results. The failure may be attributed to the possibility that the combination of values obtained were not eccentric enough to determine a well-defined elliptical orbit. The final chosen observation dates were good candidates for the Method of Gauss because the times were approximately evenly spaced out and also far apart.

The percent differences (shown in Table 10) between the mean of our calculated orbital elements and JPL Horizon's values are all below $0.02 \%$. After performing the self-consistency check between our calculated RA and DEC and the RA and DEC we observed, we found our RA values to be incredibly close, with only a $0.002 \%$ difference. However, the difference between the calculated and observed DEC had a percent difference of $0.032 \%$, which is an order of magnitude greater than the percent difference for RA.

These discrepancies were the result of many factors, particularly when it came to our data collection. When performing astrometry on our asteroid, stars and other bright objects consistently came into our asteroid's proximity. The added brightness made it more difficult for the software to calculate an accurate location of our asteroid. The 0.2 arc-second error in the AIJ plate solve found 
in our reliability checks may also have influenced the results. Furthermore, both of the telescopes we used had technical issues. The East telescope frequently drifted in the middle of our exposures and the West telescope had pointing issues, which we did try to account for. These issues made it difficult to find our asteroid, but we were eventually able to after aligning the images in AIJ.

We also chose not to include two nights of observing data. One night we were unable to find our asteroid, and the other night's data was not included because there were not enough stars in the telescope's field of view to use as references for the asteroid's position. A way that would help to improve our results would be to have more observing nights. This additional data would balance out the errors we had in some nights' data and improve the accuracy of our calculations.

Finally, the results from the numerical integrating program Swift revealed that 50 million years from now, 2002 KM6 will most likely have been ejected from the solar system or sucked into the Sun. 2002 KM6 orbits very closely to the Sun and as a result, it either travels too near or it receives a gravity assist. Table 11 summarizes the possible fates of the 60 clones of 2002 KM6.

Table 10: Fate of 2002 KM6

\begin{tabular}{lc}
\hline Percentage (\%) & Fate \\
\hline 50 & Ejected from the Solar System \\
40 & Sucked into the Sun \\
3 & Orbits too close to Saturn \\
3 & Maintains orbit \\
3 & Undetermined \\
\hline
\end{tabular}

\section{Acknowledgements}

We would like to thank Dr. Agnes Kim and Dr. Michael Dubson, the co-academic directors, for helping us throughout the research experience and providing the invaluable knowledge required to complete this project. Additionally, we thank to our four teaching assistants, Helen Cai, Bradley Emi, Julia Wei, and Jerry Xuan, for giving us support, both academically and otherwise, and especially for helping us debug our programs. Lastly, we would like to thank Fabio Mezzalira, the Operations Manager of the Sommers-Bausch Observatory at the University of Colorado Boulder, for providing us a place to work and solving all of the software and computer issues we encountered.

\section{References}

[1] Britannica, 2017, Encyclopaedia of Britannica

[2] Boulet, D. L. 1991, Richmond

[3] NASA, 2016, Our Solar System Overview 